\title{
Hipotiroidismo causando ascite e elevação de marcador tumoral/CA- 125: um relato de caso
}

\author{
Hypothyroidism causing and elevation of tumor marker/CA-125: a case report
}

Hipotiroidismo que causa ascitis y elevación del marcador de tumor/CA-125: reporte de un caso

Rudival Faial de Moraes Júnior ${ }^{1 *}$, Ricardo Paulo Pereira Mesquita², Igor Martins Gonçalves Silva², Danielle Borborema Tolentino dos Santos ${ }^{1}$, Natália Rodrigues Eugênio ${ }^{1}$, Yasaman Larissa Luyan Kós Miranda ${ }^{3}$, Pedro Arthur Ferreira de Carvalho ${ }^{3}$, Iasmin Rodrigues Salvador ${ }^{3}$.

\section{RESUMO}

Objetivo: Relatar o caso de uma paciente com ascite causada por hipotireoidismo associado com a elevação do marcador tumoral CA-125. Detalhamento do caso: Paciente feminina de 45 anos, com histórico de epilepsia secundaria a traumatismo crânio encefálico e de etilismo pesado em abstinência alcoólica há cerca de 3 meses foi encaminhada ao serviço de hepatologia da Fundação Santa Casa de Misericórdia do Pará para elucidação etiológica de ascite. Durante investigação, foram descartadas doenças hepáticas, neoplásicas e infecciosas, sendo observado $\mathrm{TSH}>100 \mathrm{Ul} / \mathrm{ml} \mathrm{com}$ ascite refratária. Após a introdução da terapia hormonal com levotiroxina em doses mais elevadas com $75 \mathrm{mcg} / \mathrm{dia}$, houve resolução do quadro clínico de ascite da paciente. Considerações finais: Este caso é notável, porque a ascite causada por hipotiroidismo é uma condição clínica rara. Sendo essa causa incomum de ascite, uma doença de fácil diagnóstico e tratamento, que deve ser pesquisada quando outras etiologias mais comuns não explicam o quadro clínico do paciente em questão.

Palavras-chave: Ascite, Hipotireoidismo, CA 125.

\begin{abstract}
Objective: To report the case of a patient with ascites caused by hypothyroidism associated with turmor marker CA-125 elevation. Case details: A 45-year-old female patient, with a history of epilepsy secondary to traumatic brain injury and heavy drinking and alcohol withdrawal for about 3 months, was referred to the hepatology service of the Fundação Santa Casa de Misericórdia do Pará for the etiological elucidation of ascites. During investigation, liver, neoplastic and infectious diseases were ruled out, and TSH $>100 \mathrm{UI} / \mathrm{ml}$ was observed with refractory ascites. After the introduction of hormonal therapy with levothyroxine at higher doses of $75 \mathrm{mcg} /$ day, the patient's clinical picture of ascites resolved. Final considerations: This case is remarkable because ascites caused by hypothyroidism is a rare clinical condition. As this uncommon cause of ascites is a disease that is easy to diagnose and treat, it should be investigated when other more common etiologies do not explain the clinical condition of the patient in question.
\end{abstract}

Keywords: Ascites, Hypothyroidism, CA 125.

\section{RESUMEN}

Objetivo: Reportar el caso de un paciente con ascitis por hipotiroidismo asociado a elevación de marcador tumoral CA-125. Detalles del caso: Una paciente de 45 años, con antecedentes de epilepsia secundaria a

\footnotetext{
${ }^{1}$ Fundação Santa Casa de Misericórdia do Pará, Belém - PA. *E-mail: rfaial@terra.com.br

${ }^{2}$ Centro Universitário do Estado do Pará (CESUPA), Belém - PA.

${ }^{3}$ Centro Universitário Metropolitano da Amazônia (FAMAZ), Belém - PA.
}

SUBMETIDO EM: 1/2022

ACEITO EM: 1/2022

PUBLICADO EM: 2/2022 
traumatismo craneoencefálico y consumo excesivo de alcohol y abstinencia de alcohol durante aproximadamente 3 meses, fue remitida al servicio de hepatología de la Fundação Santa Casa de Misericórdia do Pará para la elucidación etiológica de la ascitis. Durante la investigación se descartaron enfermedades hepáticas, neoplásicas e infecciosas y se observó TSH>100Ul / $\mathrm{ml}$ con ascitis refractaria. Después de la introducción de la terapia hormonal con levotiroxina en dosis más altas de $75 \mathrm{mcg} /$ día, el cuadro clínico de ascitis del paciente se resolvió. Consideraciones finales: Este caso es notable porque la ascitis causada por hipotiroidismo es una condición clínica rara. Dado que esta causa poco común de ascitis es una enfermedad de fácil diagnóstico y tratamiento, debe investigarse cuando otras etiologías más comunes no expliquen la condición clínica del paciente en cuestión.

Palabras clave: Ascitis, Hipotiroidismo, CA 125.

\section{INTRODUÇÃO}

O hipotiroidismo é uma disfunção da glândula tireoide que causa uma série de alterações no organismo humano, definido como um estado clínico resultante de quantidade insuficiente ou de ausência de hormônios circulantes da glândula tireoide denominados Tiroxina (T4) e Tri-iodo-tironina (T3), para suprir uma função orgânica normal (OLIVEIRA V e MALDONADO RR, 2014).

O hipotireoidismo é uma condição causada por diminuição da produção (ou ação) de hormônios tireoidianos. A expressão clínica varia de Hormônio Estimulante da Tireoide (TSH) ligeiramente elevado em pacientes assintomáticos a hipotireoidismo grave, que pode ocasionalmente causar mixedema. $O$ hipotireoidismo é classificado como primário quando há baixa atividade da glândula tireoide e representa a maioria dos casos. O hipotireoidismo central é responsável por menos de $1 \%$ dos casos e é provocado pelo acometimento da hipófise anterior (hipotireoidismo secundário) ou o acometimento do hipotálamo (hipotireoidismo terciário). Exemplos menos comuns incluem o chamado hipotireoidismo periférico, que abrange a síndrome de resistência ao hormônio da tireoide, os defeitos no metabolismo e o hipotireoidismo devido ao consumo periférico dos hormônios por tumores (RIZZO LF e MANA DL, 2020).

Esses hormônios tireoidianos circulantes em baixa quantidade ocasionam impacto negativo na taxa metabólica basal, o que repercute em diversos sistemas. Sintomas como pele seca, fadiga e intolerância ao frio são mais prevalentes. O hipotireoidismo é caracterizado pela boa resposta terapêutica a reposição hormonal com levotiroxina (MARTINELLI TC, et al., 2019).

A ascite é o acúmulo patológico de líquido na cavidade peritoneal. Como muitas doenças podem causar ascite, principalmente a cirrose, as amostras de líquido ascítico são comumente analisadas a fim de desenvolver um diagnóstico diferencial. Clinicamente, a ascite é uma consequência ou complicação de uma série de doenças, incluindo doenças hepáticas, cardíacas, renais e malignidade. A ascite geralmente carrega um prognóstico desfavorável e o seu tratamento deve ser direcionado para a sua causa base (HUANG LL, et al.,2014).

A hipertensão portal secundária à cirrose hepática é a principal causa de ascite, e o envolvimento peritoneal em pacientes com doenças malignas é o segundo. Portanto, se a composição do líquido ascítico e a ultrassonografia não forem compatíveis com a hipertensão portal ou outras doenças específicas, a malignidade deve ser considerada (KHALIL RB, et al., 2013).

A cirrose advém da elevação da pressão portal que é o principal mecanismo fisiopatológico da ascite. As células hepáticas sofrem um processo de fibrose e como consequência têm o aparecimento da ascite. Uma das complicações mais comuns da cirrose é a ascite, juntamente com a encefalopatia hepática, a síndrome hepatorrenal e a hemorragia digestiva alta (REIS SP, et al., 2019).

A ascite causada por hipotireoidismo é uma condição rara com fisiopatologia indefinida (LONGO B, et al., 2016). Menos de $4 \%$ dos pacientes com hipotireoidismo desenvolvem ascite (ABBASS A, et al., 2016). Uma análise de fluido de ascite, no contexto de hipotireoidismo, geralmente demonstra proteína elevada (>2,5g/dL) e baixa contagens de leucócitos, consistindo principalmente de linfócitos (DHINGRA R, et al., 2018). 
O câncer ovariano produz uma enzima chamada CA-125, identificada através da quimioluminescência, este exame é realizado com uma amostra sanguínea. O resultado considerado normal seria abaixo de 35 $\mathrm{U} / \mathrm{ml}$. Quando esse marcador se encontra elevado, na maioria das vezes está associado a algum tipo de câncer, majoritariamente ao câncer ovariano. Os marcadores auxiliam na triagem e podem detectar um tumor na fase inicial, e o seu diagnóstico é feito por biopsia (SOUZA PONTES N e SOARES CP, 2021).

Em alguns casos de ascite não neoplásica, como no hipotireoidismo, os níveis de CA-125 podem ser tão altos como os observados em pacientes com câncer, sugerindo que qualquer paciente com ascite e uma concentração elevada de CA-125 deve ter a função tireoidiana medida como parte de sua avaliação inicial (KHALIL RB, et al., 2013).

O objetivo do relato foi relatar o caso de uma paciente hospitalizada para investigação etiológica de um quadro de ascite, a partir de dados coletados em prontuário médico e ao mesmo tempo estabelecer uma revisão de literatura acerca dos argumentos propostos, ressaltando a importância do hipotireoidismo como diagnóstico diferencial de ascite e de origem indeterminada.

\section{ESTUDO DE CASO}

Paciente do sexo feminino, 45 anos, foi encaminhada ao serviço de referência em hepatologia no Estado do Pará, devido ao quadro de aumento do volume abdominal e ao edema de membros inferiores, com evolução de aproximadamente 4 meses.

Em avaliação inicial no dia 17 de outubro de 2019, foi observado história prévia de etilismo pesado, com abstinência de 3 meses e quadro de epilepsia aos 10 anos de idade, usando carbamazepina $200 \mathrm{mg} / \mathrm{dia}$ e fenobarbital $100 \mathrm{mg} /$ dia. $\mathrm{Na}$ ocasião a paciente trouxe laudo de Ultrassonografia (USG) de abdômen de 03/09/19, em que havia descrição de esteatose hepática severa e de ascite acentuada. Pelo exposto acima, foi levantada a hipótese de cirrose hepática descompensada e iniciado espironolactona 100mg/dia e furosemida 40mg/dia, solicitado exames laboratoriais, Tomografia Computadorizada (TC) de abdômen e Endoscopia Digestiva Alta (EDA) para complementação diagnóstica.

Devido a pandemia da Covid-19, a paciente perdeu seguimento por 10 meses, retornando a nova consulta em 14 de agosto de 2020. Durante este período foi relatada a realização de múltiplas paracenteses, além de perda ponderal de $3 \mathrm{~kg}$, abstenção alcoólica de 8 meses e redução da diurese, sem outras queixas. Dos exames solicitados anteriormente, obteve-se o resultado laboratorial de 13/11/2019 (Tabela 1).

Tabela 1 - Resultado de exames laboratoriais (13/09/2019).

\begin{tabular}{|c|c|c|c|c|c|}
\hline Exames & Resultados & Valores de referência & Exames & Resultados & $\begin{array}{l}\text { Valores de } \\
\text { referência }\end{array}$ \\
\hline Hemoglobina & $10,6 \mathrm{~g} / \mathrm{dl}$ & $(12,3-15,3 \mathrm{~g} / \mathrm{dl})$ & TGO & $63 \mathrm{U} / \mathrm{L}$ & $(15-30 U / L)$ \\
\hline Plaquetas & $521.700 / \mu \mathrm{L}$ & $\begin{array}{c}(150.000- \\
450.000 / \mu \mathrm{L})\end{array}$ & TGP & $63 \mathrm{U} / \mathrm{L}$ & $(8-35 U / L)$ \\
\hline Anti-HcV & Não reagente & $\begin{array}{l}\text { Não ser reagente se } \\
\text { não for infectado com } \\
\text { o vírus da hepatite C }\end{array}$ & Triglicerídeos & 164 mg/dl & $(<500 \mathrm{mg} / \mathrm{dl})$ \\
\hline Anti-HIV & Não reagente & $\begin{array}{l}\text { Não ser reagente se } \\
\text { não for portador do hiv }\end{array}$ & Colesterol Total & 206 mg/dl & $(<200 \mathrm{mg} / \mathrm{dl})$ \\
\hline $\mathrm{HBsAg}$ & Não reagente & Não ser reagente & CA 125 & $354 \mathrm{KU} / \mathrm{L}$ & $(<35 \mathrm{KU} / \mathrm{L})$ \\
\hline Anti-Hbs & Reagente & $\begin{array}{l}\text { Reagente se infeção } \\
\text { previa ou vacinação }\end{array}$ & Alfafetoproteína & $5,09 \mathrm{ng} / \mathrm{dl}$ & $(<15 \mathrm{ng} / \mathrm{ml})$ \\
\hline $\begin{array}{c}\text { Anti-HBc } \\
\text { Total }\end{array}$ & Reagente & Não ser reagente & TSH & $>500 \mathrm{Ul} / \mathrm{ml}$ & $\begin{array}{c}(0,34-5,6 \\
\mathrm{Ul} / \mathrm{ml})\end{array}$ \\
\hline Anti-HAV IgG & Reagente & $\begin{array}{l}\text { Ser reagente se } \\
\text { infeção prévia }\end{array}$ & T4 Livre & $0,07 \mathrm{ng} / \mathrm{dl}$ & $\begin{array}{c}(0,7- \\
1,8 \mathrm{ng} / \mathrm{dl})\end{array}$ \\
\hline CEA & $3,76 \mathrm{ng} / \mathrm{dl}$ & $(<3 n g / d l)$ & & & \\
\hline
\end{tabular}

Fonte: Júnior RFM, et al., 2022. 
No dia em questão foi solicitado novo laboratório, TC de abdômen e EDA, além da prescrição de Puran T4 25mcg/dia e encaminhamento para endocrinologista.

Em seguimento ambulatorial no dia 20/11/2020 a paciente retornou à consulta sem novas queixas, portando exames laboratoriais do dia 26/10/2020 (Tabela 2). Na TC de abdômen em 24/10/2020, foi observado fígado sem alterações e ascite acentuada. Nesse momento foi mantida posologia dos diuréticos, aumentado dose do Puran T4 para 50mcg/dia e solicitado Ressonância Magnética (RNM) de abdome superior e pelve para investigação de possível neoplasia intra-abdominal, haja vista importante elevação do marcador tumoral CA-125.

Tabela 2 - Resultado de exames laboratoriais (26/10/2020).

\begin{tabular}{cccccc}
\hline Exames & Resultados & $\begin{array}{c}\text { Valores de } \\
\text { referência }\end{array}$ & Exames & Resultados & $\begin{array}{c}\text { Valores de } \\
\text { referência }\end{array}$ \\
\hline Hemoglobina & $8,2 \mathrm{~g} / \mathrm{dl}$ & $(12,3-15,3 \mathrm{~g} / \mathrm{dl})$ & Potássio & $4,5 \mathrm{mmol} / \mathrm{L}$ & $\begin{array}{c}(3,5- \\
5,5 \mathrm{mmol} / \mathrm{L})\end{array}$ \\
\hline Plaquetas & $787.000 / \mu \mathrm{L}$ & $\begin{array}{c}(150.000- \\
450.000 / \mu \mathrm{L})\end{array}$ & TGO & $38 \mathrm{U} / \mathrm{L}$ & $(15-30 \mathrm{U} / \mathrm{L})$ \\
\hline INR & $1,1 \mathrm{isi}$ & $(0,8-1,0 \mathrm{isi})$ & TGP & $26,4 \mathrm{U} / \mathrm{L}$ & $(8-35 \mathrm{U} / \mathrm{L})$ \\
\hline Albumina & $3,3 \mathrm{~g} / \mathrm{dl}$ & $(3,2-4,5 \mathrm{~g} / \mathrm{dl})$ & Ureia & $17 \mathrm{mg} / \mathrm{dl}$ & $(16-40 \mathrm{mg} / \mathrm{dl})$ \\
\hline $\begin{array}{c}\text { Bilirrubina } \\
\text { Total }\end{array}$ & $0,25 \mathrm{mg} / \mathrm{dl}$ & $(<1,2 \mathrm{mg} / \mathrm{dl})$ & T4 Livre & $0,26 \mathrm{ng} / \mathrm{ml}$ & $(0,7-1,8 \mathrm{ng} / \mathrm{dl})$ \\
\hline $\begin{array}{c}\text { Colesterol } \\
\text { Total }\end{array}$ & $128 \mathrm{mg} / \mathrm{dl}$ & $(<200 \mathrm{mg} / \mathrm{dl})$ & TSH & $>100 \mathrm{Ul} / \mathrm{ml}$ & $(0,34-5,6$ \\
\hline Creatinina & $0,71 \mathrm{mg} / \mathrm{dl}$ & $(0,6-1,1 \mathrm{mg} / \mathrm{dl})$ & Alfafetoproteína & $6 \mathrm{ng} / \mathrm{ml}$ & $(<15 \mathrm{ng} / \mathrm{ml})$ \\
\hline $\begin{array}{c}\text { Fosfatase } \\
\text { Alcalina }\end{array}$ & $91 \mathrm{U} / \mathrm{L}$ & $(46-120 \mathrm{U} / \mathrm{L})$ & CA 125 & $969 \mathrm{KU} / \mathrm{L}$ & $(<35 \mathrm{KU} / \mathrm{L})$ \\
\hline Gama GT & $50 \mathrm{U} / \mathrm{L}$ & $(7-50 \mathrm{U} / \mathrm{L})$ & C19-9 & $4,7 \mathrm{U} / \mathrm{ml}$ & $(<37 \mathrm{U} / \mathrm{ml})$ \\
\hline Glicose & $89 \mathrm{mg} / \mathrm{dl}$ & $(70-99 \mathrm{mg} / \mathrm{dl})$ & CEA & $2,6 \mathrm{mcg} / \mathrm{L}$ & $(<3 \mathrm{mcg} / \mathrm{L})$ \\
\hline Sódio & $137 \mathrm{mmol} / \mathrm{L}$ & $(135-145 \mathrm{mmol} / \mathrm{L})$ & & & \\
\hline Fon & & &
\end{tabular}

Fonte: Júnior RFM, et al.,2022.

Paciente permaneceu estável no período interconsulta, voltando ao ambulatório em 12/01/2021 para dar continuidade a investigação etiológica da ascite e a possibilidade de doença neoplásica. $\mathrm{Na}$ ocasião foi confirmado por RNM de abdome superior e pelve (09/12/2020) a presença de fígado com características normais, ascite acentuada, não visibilização dos ovários, linfonodos negativos, sem metástases ou outros focos em evidência e útero de pequeno tamanho. Em EDA (27/11/2020) foi observado gastrite enantematosa leve de antro, sem descrição de varizes esofágicas. Como visto acima, apesar da extensa investigação ambulatorial, não se obteve resultados que pudessem fornecer confirmação diagnóstica e com a elevação do marcador tumoral CA-125, optou-se por solicitar internação hospitalar.

Paciente foi admitida em 14/01/2021 na enfermaria de Clínica Médica para acompanhamento com as equipes de hepatologia, de ginecologia e obstetrícia e de clínica médica da instituição. Nesse momento, sob supervisão da equipe de hepatologia, as impressões diagnósticas eram: ascite a esclarecer, hipotireoidismo, anemia com discreta plaquetose. Na evolução diária referia desconforto abdominal devido a ascite. Ao exame físico: bom estado geral, consciente e orientada, sem alterações ao exame físico, exceto por abdome globoso, normotenso, com presença de macicez móvel. No momento da internação foram suspensos diuréticos pela falta de evidencias de ascite por causa hepática, e foram mantidas medicações antiepilépticas e Puran T4 $50 \mathrm{mcg}$.

Nos exames laboratoriais da admissão foram visibilizadas alterações semelhantes ao já exposto acima. Foram então solicitados novos exames complementares (Tabela 3 e Tabela 4). 
Tabela 3 - Resultado de exames laboratoriais (15/01/2021).

\begin{tabular}{cccccc}
\hline Exames & Resultados & $\begin{array}{c}\text { Valores de } \\
\text { referência }\end{array}$ & Exames & Resultados & $\begin{array}{c}\text { Valores de } \\
\text { referência }\end{array}$ \\
\hline Albumina & $4,4 \mathrm{~g} / \mathrm{dl}$ & $(3,2-4,5 \mathrm{~g} / \mathrm{dl})$ & T4 Livre & $0,33 \mathrm{ng} / \mathrm{ml}$ & $(0,7-1,8 \mathrm{ng} / \mathrm{dl})$ \\
\hline Ferro sérico & $27 \mathrm{micromol} / \mathrm{L}$ & $(11-25 \mathrm{micromol} / \mathrm{L})$ & TSH & $>100 \mathrm{Ul} / \mathrm{ml}$ & $(0,34-5,6 \mathrm{Ul} / \mathrm{ml})$ \\
\hline $\begin{array}{c}\text { Colesterol } \\
\text { Total }\end{array}$ & $186 \mathrm{mg} / \mathrm{dl}$ & $(<200 \mathrm{mg} / \mathrm{dl})$ & Ácido Fólico & $5,3 \mathrm{ng} / \mathrm{ml}$ & $(3,10-20,5 \mathrm{ng} / \mathrm{ml})$ \\
\hline LDL & $78,9 \mathrm{mg} / \mathrm{dl}$ & $(<160 \mathrm{mg} / \mathrm{dl})$ & Anti-TPO & $1,5 \mathrm{U} / \mathrm{ml}$ & $(<20 \mathrm{U} / \mathrm{ml})$ \\
\hline Triglicerídeos & $98 \mathrm{mg} / \mathrm{dl}$ & $(<500 \mathrm{mg} / \mathrm{dl})$ & TRAB & $0,1 \mathrm{U} / \mathrm{ml}$ & $(<1,75 \mathrm{U} / \mathrm{ml})$ \\
\hline Glicose & $92 \mathrm{mg} / \mathrm{dl}$ & $(<100 \mathrm{mg} / \mathrm{dl})$ & VSH & $110 \mathrm{~mm} / \mathrm{h}$ & $(<25 \mathrm{~mm} / \mathrm{h})$ \\
\hline Ferritina & $42,7 \mathrm{ng} / \mathrm{ml}$ & $(11-206 \mathrm{ng} / \mathrm{ml})$ & PCR & $60 \mathrm{mg} / \mathrm{l}$ & $(<10 \mathrm{mg} / \mathrm{l})$ \\
\hline
\end{tabular}

Fonte: Júnior RFM, et al., 2022.

Tabela 4 - Resultado da análise do líquido ascítico (15/01/2021).

\begin{tabular}{cccccc}
\hline Exames & Resultados & $\begin{array}{c}\text { Valores de } \\
\text { referência }\end{array}$ & Exames & Resultados & $\begin{array}{c}\text { Valores de } \\
\text { referência }\end{array}$ \\
\hline Cor & Amarelo & (amarelo) & Celularidade & 4.160 & Sem parâmetro \\
\hline GASA & 0,1 & $\begin{array}{c}(>1,1 \text { é hipertensão } \\
\text { portal ou }<1,1 \text { é } \\
\text { exudato })\end{array}$ & Hemácias & $4.480 / \mu \mathrm{L}$ & $(<100.000 / \mu \mathrm{L})$ \\
\hline Amilase & $2.221 \mathrm{U} / \mathrm{L}$ & $(20-100 \mathrm{U} / \mathrm{L})$ & Linfócitos & $19 \%$ & Sem parâmetro \\
\hline DHL & $555 \mathrm{U} / \mathrm{l}$ & $(<200 \mathrm{U} / \mathrm{l})$ & $\begin{array}{c}\text { Células } \\
\text { Mesoteliais }\end{array}$ & $73 \%$ & Sem parâmetro \\
\hline Glicose & $100 \mathrm{mg} / \mathrm{d}$ & $(70-100 \mathrm{mg} / \mathrm{dl})$ & $\begin{array}{c}\text { Células } \\
\text { atípicas }\end{array}$ & Ausentes & Ser ausente \\
\hline Lipase & $22 \mathrm{UI} / \mathrm{L}$ & $(13-60 \mathrm{UI} / \mathrm{L})$ & $\begin{array}{c}\text { Proteínas } \\
\text { Totais }\end{array}$ & $0,15 \mathrm{~g} / \mathrm{dl}$ & Sem parâmetro \\
\hline
\end{tabular}

Fonte: Júnior RFM, et al., 2022.

Em 18/01/21 foi avaliada pela equipe de Ginecologia e Obstetrícia da instituição, que ao exame físico não identificou alterações. Nesse momento foram solicitados exames laboratoriais hormonais e USG Transvaginal para descartar doenças neoplásicas ovarianas germinativas.

No dia 19/01/2021 foram solicitados novos exames laboratoriais com os mesmos parâmetros e sem informações relevantes. RNM de abdome total com contraste: pequena quantidade de liquido em cavidade abdominal. Dessa forma, recebeu alta da equipe de hepatologia e foi conduzida a receber supervisão da clínica médica.

Dia 20/01/2021, no $7^{\circ}$ dia de internação, já sob supervisão da clínica médica, permanecia com os mesmos problemas ativos: ascite a esclarecer, hipotireoidismo e anemia com discreta plaquetose. Na evolução diária ainda relatava desconforto abdominal devido a ascite. Ao exame físico encontrava-se com os mesmos achados. A conduta foi o ajuste da dose de Puran T4 para $75 \mathrm{mcg}$. No dia 21/01/2021, a paciente referiu leve melhora do desconforto ocasionado pela ascite e no mesmo dia foi reavaliada pela Ginecologia sem informações dignas de nota com resultado dos exames anteriormente requisitados sem alterações, recebendo alta desta equipe.

Dia 26/01/2021 a paciente relatou melhora gradativa e significativa do desconforto abdominal. Nessa avaliação foi solicitado uma colonoscopia para investigação complementar.

No $19^{\circ}$ dia de internação hospitalar, em 01/02/2021, sob supervisão da equipe de clínica médica, com resultado da colonoscopia (29/01/2021) sem alterações, teve alta hospitalar com resolução do quadro de ascite. Foram mantidos Puran T4 75mcg, medicações antiepilépticas e encaminhada para acompanhamento ambulatorial com a clínica médica.

Este relato foi aprovado pelo Comitê de Ética e Pesquisa (CEP) com o CAAE: 46653821.3.0000.5171. 


\section{DISCUSSÃO}

A ascite causada por hipotireoidismo é uma condição rara com fisiopatologia indefinida (LONGO B, et al., 2016). Sendo necessárias publicações científicas que corroborem essa etiologia no leque de diagnósticos diferenciais da comunidade médica. Pela singularidade dessa condição clínica é frequentemente confundida com outras doenças.

Inicialmente foi feita à exclusão dos principais diagnósticos relacionados a ascite durante o período de acompanhamento da paciente no hospital. Relacionadas as causas infecciosas, paciente teve marcadores negativos (HBsAg, Anti-HCV) de hepatites virais durante a internação, excluindo os diagnósticos diferenciais de hepatites B e C (SILVA AL, et al., 2012). Somente o Anti-HAV IgG estava reagente, indicando que a paciente teve infecção passada ou foi vacinada, da mesma forma que a infecção pelo HVA é geralmente autolimitada e dificilmente provoca hepatopatia crônica (ARANTES MJ, 2020).

Além disso, os exames laboratoriais de função hepática estavam dentro dos limites da normalidade, assim como todas as RNMs e TCs, feitas na FSCMP, não demonstraram alterações hepáticas, descartando cirrose devido não ter evidências de processo hepático extenso, caracterizado por fibrose e nódulos estruturalmente anormais (REIS SP, et al.,2019). Ademais outro fator nos exames complementares com fator preditivo negativo para cirrose foi a ausência de plaquetopenia que é um achado muito comum na cirrose, sendo que a paciente apresentava plaquetose. Dessa forma, por todos os fatores supracitados foi descartada a cirrose como hipótese diagnóstica (SOUZA EO, 2017).

O único exame de imagem que teve alteração foi a USG, realizada em instituição privada anteriormente ao acompanhamento na FSCMP. Nesse exame foi constatado esteatose hepática. Vale ressaltar que USG é o método diagnóstico mais simples para a esteatose hepática, quando está é maior que 30\% (CRUZ JF, et al., 2016). Porém, é importante mencionar que a USG é um exame operador-dependente, ao contrário das TCs e RNMs que não apresentaram alterações.

Seguindo a linha de raciocínio clínico de comprometimento hepático, um dos diagnósticos diferenciais é a doença hepática gordurosa não alcoólica (DHGNA). A DHGNA inclui, em seu espectro, desde esteatose simples e apenas acúmulo de gordura no fígado, até esteato-hepatite com componente necroinflamatório, cirrose e carcinoma hepatocelular (CRUZ JF, et al., 2016). Essa condição tem como principal fator de risco doenças responsáveis por distúrbios metabólicos, como obesidade, diabetes mellitus tipo II e hiperlipidemias, e outras comorbidades que lesem os hepatócitos, como hepatite $C$ e uso de drogas e álcool (SILVA LDCMD, et al., 2019). A paciente em questão não apresentava nenhuma das condições acima, exceto consumo de álcool.

Ademais, importante salientar que em agosto de 2020 relatava abstinência alcoólica há 8 meses, sem melhora do quadro clínico da ascite, sendo isso fator preditivo negativo para hepatite/cirrose alcoólica. Esse diagnóstico foi excluído por não apresentar alterações nos exames de imagem e laboratoriais.

A elevação do marcador tumoral CA-125 levantou grande suspeita de neoplasia como causa da ascite. Os marcadores tumorais vieram com a função de identificar o câncer de ovário em mulheres assintomáticas, presumindo-se que teriam o poder de sinalizar o tumor em fase inicial, associado a USG com presença de massa ovariana, para confirmar a hipótese e identificar risco de malignidade tumoral (SOUZA PONTES $\mathrm{N} \mathrm{e}$ SOARES CP, 2021). Essa questão foi motivo de cuidadosa e extensa investigação das equipes de Clínica Médica, Ginecologia e Obstetrícia e Hepatologia da referida instituição.

A equipe de GO realizou investigação completa com exame físico ginecológico normal, exames laboratoriais dos hormônios sexuais dentro dos níveis de normalidade, tendo como única alteração na RNM de pelve grande quantidade de líquido. Desse modo foi excluído a hipótese diagnóstica de neoplasia ginecológica pois o biomarcador sérico CA-125 é incapaz de confirmar diagnóstico sozinho, pois se altera com outras doenças benignas (SOUZA PONTES N e SOARES CP, 2021).

Corrobora com essa linha de raciocínio o entendimento que a USG está entre os principais métodos para diagnosticar o câncer ovariano. A Ultrassonografia se destaca ao avaliar e caracterizar massas císticas simples, anexiais sólidas ou anexiais complexas. As massas anexiais sólidas podem ser benignas ou malignas, as quais incluem os teratomas císticos, fibromas, e câncer ovariano (RODRIGUES GJR, et al., 2021). Nenhuma dessas evidências foi encontrada no USG da paciente em questão. 
Em alguns casos de ascite não neoplásicas, como a ascite mixedematosa, os níveis de CA-125 podem estar tão elevados como os observados em pacientes com câncer, sugerindo que todos paciente com ascite e uma concentração elevada de CA-125 deve ter a função tireoidiana medida como parte de sua avaliação inicial como realizado neste relato de caso. Normalmente, esses pacientes são submetidos a terapia diurética, laparotomias diagnósticas e paracentese até que as causas com maior potencial de gravidade sejam afastadas (KHALIL RB, et al., 2013). Acredita-se que este marcador esteja elevado devido a irritação peritoneal causada pela ascite ou pelo próprio hipotiroidismo (SOUZA PONTES N e SOARES CP, 2021).

Durante o início do seu acompanhamento no ambulatório de hepatologia foi diagnosticada com hipotireoidismo com TSH > $500 \mathrm{Ul} / \mathrm{ml}$, sendo iniciado Puran T4 $25 \mathrm{mcg}$. Posteriormente, a dose do medicamento foi aumentada para $50 \mathrm{mcg}$, pois o TSH ainda não se encontrava dentro dos valores de referência. Concomitante a essa terapêutica, ocorria a internação e investigação pelas equipes médicas da instituição, pois a paciente permanecia sem melhora do quadro de ascite. Durante seu período de internação em janeiro de 2021, a dose do Puran T4 foi otimizada para $75 \mathrm{mcg}$. Nos dias subsequentes relatou melhora progressiva da ascite, até sua completa resolução próximo à alta hospitalar. Dessa forma, a equipe médica fez por exclusão, o diagnóstico de ascite mixedematosa, devido a melhora clínica após a otimização da dose de levotiroxina (DANTAS RKN, et al., 2021).

Neste relato de caso, através da prova terapêutica com levotiroxina, foi confirmado a relação entre ascite gerada por hipotireoidismo e elevação do CA-125. Concluímos que o hipotireoidismo deve ser incluído como diagnóstico diferencial de ascite sem causa aparente, em que a reposição do hormônio tireoidiano se mostra uma conduta resolutiva. Portanto, é fundamental destacar que após a exclusão das principais hipóteses ginecológicas, hepáticas e neoplásicas e a evidente melhora clínica da paciente com aumento da dose de levotiroxina, a equipe médica realizou o diagnóstico de ascite mixedematosa causada por hipotireoidismo e associada a elevação do marcador tumoral CA -125.

\section{REFERÊNCIAS}

1. ABBASS A, et al. The Curious Case of a Swollen Belly: Myxedema Ascites: 2236. Official journal of the American College of Gastroenterology| ACG,2016; 111: S1075.

2. ARANTES MJ. Resposta sorológica à vacinação ou infecção pela hepatite $A$ em crianças e adolescentes infectados pelo HIV. Dissertação (Trabalho de Conclusão de Curso em Medicina) - Centro de Ciências e Saúde. Universidade Federal de Santa Catarina, 2020; 10-13 p.

3. CRUZ JF, et al. Relação entre a esteatose hepática não alcoólica e as alterações dos componentes da síndrome metabólica e resistência à insulina. Revista da Sociedade Brasileira de Clínica Médica, $2016 ; 14$ (2): 79-83.

4. SILVA AL, et al. Hepatites virais: B, C e D: atualização. Revista da Sociedade Brasileira de Clínica Médica, 2012.10(3): 206-18.

5. DANTAS RKN, et al. Coma Mixedematoso em paciente com síndrome do Eutireoideo doente: um relato de caso. Brazilian Journal of Health Review, 2021; 4(3): 9868-9877.

6. SOUZA PONTES N, SOARES CP. Marcadores tumorais específicos para câncer de ovário CA

7. DHINGRA R, et al. Myxedema Ascites: An Unusual Presentation of Uncontrolled Hypothyroidism. Cureus, 2018;10(5).

8. HUANG LL, et al. Ascitic fluid analysis in the differential diagnosis of ascites: focus on cirrhotic ascites. Journal of clinical and translational hepatology, 2014; 2 (1): 58.

9. KHALIL RB, et al. Myxedema ascites with high CA-125: case and a review of literature. World journal of hepatology, 2013; 5(2): 86.

10. LONGO B, et al. UMA CAUSA INCOMUM DE ASCITE. Revista Médica UFPR, 2016; 3(2).

11. MARTINELLI TC, et al. Cardiomegalia associada ao hipotireoidismo clínico. Revista de Medicina, 2019; 98(1): 81-85.

12. OLIVEIRA V, MALDONADO RR. Hipotireoidismo e hipertireoidismo - Uma breve revisão sobre as disfunções tireoidianas. Interciência \& Sociedade, 2014; 3 (2).

13. REIS SP, et al. Ascite: complicação da cirrose. Cadernos da Medicina-UNIFESO, $2019 ; 2$ (1).

14. RIZZO LF, MANA DL. Treatment of hypothyroidism in special situations. MEDICINA (Buenos Aires), 2020; 80:83- 93.

15. RODRIGUES GJR, et al. A relevância da implementação de um programa de rastreamento de câncer de ovário: uma revisão narrativa. Revista Eletrônica Acervo Saúde, 2021;13(9): e8390.

16. SILVA LDCMD, et al. Análise da elastografia por ultrassonografia em pacientes com esteatose hepática. Radiologia Brasileira, 2019; 53: 47-55.

17. SOUZA EO. Avaliação funcional das plaquetas em pacientes com cirrose e sua relação com o risco de sangramento após ligadura elástica de varizes esofagianas. Dissertação (Doutorado em Ciências) - Programa de Ciências em Gastroenterologia. Universidade de São Paulo, 2017; 3 p. 